Trinity University

Digital Commons@ Trinity

Mathematics Faculty Research

Mathematics Department

6-2002

\title{
Global Stability of Cycles: Lotka-Volterra Competition Model with Stocking
}

Saber Elaydi

Trinity University, selaydi@trinity.edu

Abdul-Aziz Yakubu

Follow this and additional works at: https://digitalcommons.trinity.edu/math_faculty

Part of the Mathematics Commons

\section{Repository Citation}

Elaydi, S., \& Yakubu, A.-A. (2002). Global stability of cycles: Lotka-Volterra competition model with stocking. Journal of Difference Equations and Applications, 8(6), 537-549. doi:10.1080/10236190290027666

This Post-Print is brought to you for free and open access by the Mathematics Department at Digital Commons @ Trinity. It has been accepted for inclusion in Mathematics Faculty Research by an authorized administrator of Digital Commons @ Trinity. For more information, please contact jcostanz@trinity.edu. 


\title{
GLOBAL STABILITY OF CYCLES: LOTKA-VOLTERRA COMPETITION MODEL WITH STOCKING
}

\author{
Saber Elaydi \\ Department of Mathematics \\ Trinity University \\ San Antonio, Texas \\ and \\ Abdul-Aziz Yakubu \\ Department of Mathematics \\ Howard University \\ Washington, DC 20059
}

April 27, 2001

\begin{abstract}
In this article, we prove that in connected metric spaces $k$-cycles are not globally attracting (where $k \geq 2$ ). We apply this result to a two species discrete-time Lotka-Volterra competition model with stocking. In particular, we show that an $k$-cycle cannot be the ultimate life-history of evolution of all population sizes. This solves Yakubu's conjecture but the question on the structure of the boundary of the basins of attraction of the locally stable $n$-cycles is still open.
\end{abstract}

Keywords: global attractivity, cycles, Lotka Volterra, competition model, stocking. 
AMS Mathematical Subject Classification: 39A10, 39A11.

\section{INTRODUCTION}

Mathematical models have provided important insights into the general conditions that permit the coexistence of competing species and the circumstances that lead to competitive exclusion [1, 2, 4, 6-18, 20-29]. The following mathematical model, System (1), describes the growth dynamics of two species (Species 1 and 2) in competition, where both species are governed by Ricker's model and Species 1 is being stocked at the constant per capita stocking rate $\alpha$ per generation [28, 29]:

$$
\left.\begin{array}{lcc}
x_{1}(t+1)= & x_{1}(t) \exp \left(p_{1}-q_{1}\left(x_{1}(t)+x_{2}(t)\right)\right)+\alpha x_{1}(t), \\
x_{2}(t+1)= & x_{2}(t) \exp \left(p_{2}-q_{2}\left(x_{1}(t)+x_{2}(t)\right)\right) .
\end{array}\right\}
$$

For each Species $i \in\{1,2\}, x_{i}(t)$ is its population size at generation $t$ and $p_{i}, q_{i}$ and $\alpha$ are positive constants. The effects of population density on the survival and growth of each individual species are modeled in System (1), by assuming that each per-capita growth rate, $g_{i}\left(x_{1}+x_{2}\right)=\exp \left(p_{i}-q_{i}\left(x_{1}+x_{2}\right)\right)$, is a function of the total density of the two competitors. For species with closely spaced generations, System (1) without stocking reduces to the Lotka-Volterra differential equations [16]. Notice that System (1) has no isolated positive fixed points.

If the carrying capacity of Species $1, \frac{p_{1}}{q_{1}}$, is less than that of Species $2, \frac{p_{2}}{q_{2}}$, then Species 1 goes extinct whenever there is no stocking $(\alpha=0)$ [10]. Consequently, $\frac{p_{1}}{q_{1}}<\frac{p_{2}}{q_{2}}$ implies that the endangered Species 1 is the only species being stocked. Very small values of the constant per capita stocking rate $\alpha$ do not save the endangered species from extinction. However, it is possible for the endangered species without stocking to become the dominant species with stocking [28]. This reverse exclusion principle occurs whenever the constant per capita rate of stocking $\alpha$ is sufficiently large. Intermediate values of $\alpha$ promote the stable coexistence of the two competitors via the emergence of stable $k$-cycles. The boundary of 
the basin of attraction of the $k$-cycles can be fractal in nature [28, 29].

Yakubu obtained parameter regimes for the occurrence of an attracting 2cycle in System (1) and conjectured on the existence of globally stable 2-cycles $[20,21,28,29]$. In this paper, we solve Yakubu's problem and we prove that in connected metric spaces, $k$-cycles are not globally attracting. Therefore, in System (1), an $k$-cycle cannot be the ultimate life-history of evolution of all population sizes.

\section{NOTATIONS AND PRELIMINARIES}

To write the reproduction function of System (1), we denote the vector of population densities $x(t)=\left(x_{1}(t), x_{2}(t)\right)$ by $x=\left(x_{1}, x_{2}\right)$ and define the map

$$
F:[0, \infty) \times[0, \infty) \rightarrow[0, \infty) \times[0, \infty)
$$

by

$$
F\left(x_{1}, x_{2}\right)=\left(x_{1} g_{1}\left(x_{1}+x_{2}\right)+\alpha x_{1}, x_{2} g_{2}\left(x_{1}+x_{2}\right)\right)
$$

where the variable planting coefficient $\alpha$ is positive and where $g_{i}\left(x_{i}\right)=\exp \left(p_{i}-\right.$ $\left.q_{i} x_{i}\right)$ for each $i \in\{1,2\} . F^{t}$ is the map $F$ composed with itself $t$ times, and $F_{j}^{t}(x)$ is the $j^{t h}$ component of $F^{t}$ evaluated at the point $x=\left(x_{1}, x_{2}\right)$ in $\mathbb{R}_{+}^{2}$. Therefore, $F^{t}$ gives the population densities in generation $t$. The set of iterates of the map $F$ is equivalent to the set of all density sequences generated by System (1).

Define the single species, 1-dimensional maps $f_{1}: \mathbb{R}_{+} \rightarrow \mathbb{R}_{+}$and $f_{2}: \mathbb{R}_{+} \rightarrow$ $\mathbb{R}_{+}$by $f_{1}\left(x_{1}\right)=x_{1} \exp \left(p_{1}-q_{1} x_{1}\right)+\alpha x_{1}$ and $f_{2}\left(x_{2}\right)=x_{2} \exp \left(p_{2}-q_{2} x_{2}\right)$, respectively. If Species $j$ is missing in System (1), then $F$ reduces to $f_{i}$, where $i \neq j \in\{1,2\}$. The only positive fixed point of $f_{1}$ is $X_{1} \equiv \frac{p_{1}-\ln (1-\alpha)}{q_{1}}$ while that of $f_{2}$ is $X_{2} \equiv \frac{p_{2}}{q_{2}}$. Consequently, $\left(\frac{p_{1}-\ln (1-\alpha)}{q_{1}}, 0\right)$ and $\left(0, \frac{p_{2}}{q_{2}}\right)$ are the non-zero boundary fixed points of $F$.

Since $g_{i}$ is a strictly decreasing continuous function, $f_{i}\left(x_{i}\right)>x_{i}$ whenever $0<x_{i}<X_{i}$ and $f_{i}\left(x_{i}\right)<x_{i}$ whenever $x_{i}>X_{i}$. Consequently, under $f_{i}$ iterations, 
$I_{i} \equiv f_{i}\left(\left[0, X_{i}\right]\right)$ is a compact invariant interval in $\mathbb{R}_{+}$. Every point either eventually enters $I_{i}$ and stays or just reaches a limit in it. Thus, $I_{i} \equiv f_{i}\left(\left[0, X_{i}\right]\right)$ is a global attractor under $f_{i}$ iterations. In fact, under $f_{i}$ iterations, $I_{i}$ is the dual attractor to infinity. Notice that the fixed point zero in $I_{i}$ is a repellor. The largest attractor in the open interval $(0, \infty)$, denoted by $T_{i}$, is the dual attractor to the pair of repellors zero and infinity (under $f_{i}$ iterations) [9]. In [28], Yakubu showed that when

$$
p_{1}<\frac{2}{(1-\alpha)}+\ln (1-\alpha)
$$

then

$$
T_{1}=\left\{\frac{p_{1}-\ln (1-\alpha)}{q_{1}}\right\}
$$

and, when

$$
p_{2} \in(0,2),
$$

then

$$
T_{2}=\left\{\frac{p_{2}}{q_{2}}\right\}
$$

Large values of $p_{1}$ and $p_{2}$ give rise to complex (chaotic) dynamics on the attractors $T_{1}$ and $T_{2}$, respectively. We will use the following dominance criteria of Franke and Yakubu [9]:

Species 1 dominates 2 whenever $\max T_{1}<\min T_{2}$,

and,

Species 2 dominates 1 whenever $\max T_{2}<\min T_{1}$.

A general result of Franke and Yakubu implies that the dominant species drives the dominated species to extinction in System (1) [9]. In fact, using this result we obtain the following:

Theorem 1: $X_{2} \equiv \frac{p_{2}}{q_{2}}$ is a global attractor on the 2 - axis and Species 2 drives 1 to extinction whenever inequalities (1) and (2) hold and $X_{1}<X_{2}$. 
Theorem 2: $X_{1} \equiv \frac{p_{1}-\ln (1-\alpha)}{q_{1}}$ is a global attractor on the 1 -axis and Species 1 drives 2 to extinction whenever inequalities (1) and (2) hold and $X_{1}>X_{2}$.

Theorem 1 and Theorem 2 complement Theorem 5 in [28]. In Theorem 5, cited above, it is assumed that $q_{1}=q_{2}$ and $e^{p_{2}}-e^{p_{1}}<\alpha$. Then from Theorem 1 we have $p_{1}-\ln (1-\alpha)<p_{2}$. Exponentiating both sides yields, $e^{p_{1}}<(1-\alpha) e^{p_{2}}<$ $(1-\alpha)\left(\alpha+e^{p_{1}}\right)$. Hence, $p_{1}<\ln (1-\alpha)<0$ a contradiction.

In [28], Yakubu proved that there is no population explosion in System (1). Hence no point has an unbounded orbit. To understand the properties of System $(1)$, we describe regions in $[0, \infty) \times[0, \infty)$ where each species increases or decreases in abundance.

If $x_{1}+x_{2}<X_{i}$ then $F_{i}\left(x_{1}, x_{2}\right)>x_{i}$, and, if $x_{1}+x_{2}>X_{i}$ then $F_{i}\left(x_{1}, x_{2}\right)<x_{i}$. That is, after one generation the population size of Species $i$ increases [respectively, decreases] whenever the total population is smaller [respectively, bigger] than its carrying capacity. Consequently, we divide the first quadrant into three regions based on whether the first coordinate increases or decreases under $F$ iterations.

Whenever $X_{1}<X_{2}$, we let $A \equiv\left\{\left(x_{1}, x_{2}\right) \in[0, \infty) \times[0, \infty) \mid x_{1}+x_{2}>\right.$ $\left.X_{2}\right\}, B \equiv\left\{\left(x_{1}, x_{2}\right) \in[0, \infty) \times[0, \infty) \mid x_{1}+x_{2}<X_{1}\right\}$ and $M \equiv\left\{\left(x_{1}, x_{2}\right) \in\right.$ $\left.[0, \infty) \times[0, \infty) \mid X_{1}<x_{1}+x_{2}<X_{2}\right\}$. In region $A$ [respectively, $\left.B\right]$, both coordinates of points decrease [respectively, increase] under $F$ iteration and population sizes of Species 1 and 2 decrease [respectively, increase]. On the line segment $L \equiv\left\{\left(x_{1}, x_{2}\right) \in[0, \infty) \times[0, \infty) \mid x_{1}+x_{2}=X_{1}\right\}$, after one generation, the first coordinate remains the same while the second coordinate increases under $F$ iteration. That is, if a population size is on $L$, after one generation, the population size of Species 2 increases while that of Species 1 remains the same. In region $M$, after one generation, the first coordinate decreases while the second coordinate increases under $F$ iteration, that is, the population size of Species 1 decreases 
while that of Species 2 increases.

\section{Boundary Fixed Points}

The one-hump single species models, $f_{1}$ and $f_{2}$, describe the dynamics on the axes and are capable of supporting period-doubling bifurcations including complex (chaotic) dynamics. In this section, we use $f_{1}$ and $f_{2}$ to study the ultimate life-history evolution of populations under $F$ iterations.

We need the following result on the (local) stability of the boundary fixed points and periodic points.

Lemma 1: The boundary fixed points are $(0,0), X_{1}$ and $X_{2}$.

1. The unstable fixed point $(0,0)$ has unstable manifolds on the 1 - axis and 2 -axis.

2. If $X_{1}<X_{2}$ then $X_{2}$ has a stable manifold in the interior of $R_{+}^{2}$ while $X_{1}$ has an unstable manifold in the interior of $R_{+}^{2}$.

3. If $X_{1}>X_{2}$ then $X_{1}$ has a stable manifold in the interior of $R_{+}^{2}$ while $X_{2}$ has an unstable manifold in the interior of $R_{+}^{2}$.

4. If $p_{2}<2$, then $X_{2}$ has a stable manifold on the 2 -axis. As $p_{2}$ increases past 2, a period-doubling bifurcation occurs resulting in the birth of a stable 2 - cycle with stable manifold on the 2 - axis. Further increases in $p_{2}$ values with all other parameters fixed generate stable $2^{n}$-cycles (where $n \in\{2,3, \ldots\})$ with stable manifold on the 2 - axis.

5. If $p_{1}<\frac{2}{(1-\alpha)}+\ln (1-\alpha)$, then $X_{1}$ has a stable manifold on the 1 -axis. As $p_{1}$ increases past $\frac{2}{(1-\alpha)}+\ln (1-\alpha)$, a period-doubling bifurcation occurs resulting in the birth of a stable 2-cycle with stable manifold on the 1-axis. Further increases in $p_{1}$ values with all other parameters fixed generate stable $2^{n}$ - cycles (where $\left.n \in\{2,3, \ldots\}\right)$ with stable manifold on the 1 - axis. 
To prove (1), (2) and (3) we compute the Jacobian matrix

$$
D F\left(x_{1}, x_{2}\right)=\left(\begin{array}{cc}
\left(1-q_{1} x_{1}\right) \exp \left(p_{1}-q_{1}\left(x_{1}+x_{2}\right)\right)+\alpha & -q_{1} x_{1} \exp \left(p_{1}-q_{1}\left(x_{1}+x_{2}\right)\right) \\
-q_{2} x_{2} \exp \left(p_{2}-q_{2}\left(x_{1}+x_{2}\right)\right) & \left(1-q_{2} x_{2}\right) \exp \left(p_{2}-q_{2}\left(x_{1}+x_{2}\right)\right)
\end{array}\right) .
$$

Hence,

$$
\begin{gathered}
D F(0,0)=\left(\begin{array}{cc}
\exp \left(p_{1}\right)+\alpha & 0 \\
0 & \exp \left(p_{2}\right)
\end{array}\right) \\
D F\left(X_{1}\right)=\left(\begin{array}{cc}
1-\left(p_{1}-\ln (1-\alpha)\right)(1-\alpha) & -\left(p_{1}-\ln (1-\alpha)\right)(1-\alpha) \\
0 & \exp \left(p_{2}-q_{2} \frac{\left(p_{1}-\ln (1-\alpha)\right)}{q_{1}}\right)
\end{array}\right)
\end{gathered}
$$

and,

$$
D F\left(X_{2}\right)=\left(\begin{array}{cc}
\exp \left(p_{1}-q_{1} \frac{p_{2}}{q_{2}}\right)+\alpha & 0 \\
-p_{2} & 1-p_{2}
\end{array}\right),
$$

$D F(0,0)$ has eigenvalues $\lambda_{1}=\exp \left(p_{1}\right)+\alpha>1$ and $\lambda_{2}=\exp \left(p_{2}\right)>1$ with corresponding eigenvectors $v_{1}=\left(\begin{array}{l}1 \\ 0\end{array}\right)$ and $v_{2}=\left(\begin{array}{l}0 \\ 1\end{array}\right)$, and this proves (1). $D F\left(X_{1}\right)$ has eigenvalues $\lambda_{1}=1-\left(p_{1}-\ln (1-\alpha)\right)(1-\alpha)$ and $\lambda_{2}=\exp \left(q_{2}\left(X_{2}-X_{1}\right)\right)$ with corresponding eigenvectors

$$
v_{1}=\left(\begin{array}{l}
1 \\
0
\end{array}\right)
$$

and

$$
v_{2}=\left(\begin{array}{c}
\left(p_{1}-\ln (1-\alpha)\right)(1-\alpha) \\
1-\left(p_{1}-\ln (1-\alpha)\right)(1-\alpha)-\exp \left(p_{2}-q_{2} \frac{\left(p_{1}-\ln (1-\alpha)\right)}{q_{1}}\right)
\end{array}\right)
$$

while $D F\left(X_{2}\right)$ has eigenvalues $\lambda_{1}=1-p_{2}$ and $\lambda_{2}=\exp \left(p_{1}-q_{1} X_{2}\right)+\alpha$ with corresponding eigenvectors $v_{1}=\left(\begin{array}{l}0 \\ 1\end{array}\right)$ and $v_{2}=\left(\begin{array}{c}1-p_{2}-\left(\exp \left(p_{1}-q_{1} \frac{p_{2}}{q_{2}}\right)+\alpha\right) \\ p_{2}\end{array}\right)$. $X_{1}<X_{2}$ implies that the external eigenvalue of $D F\left(X_{1}\right), \lambda_{2}=\exp \left(q_{2}\left(X_{2}-\right.\right.$ $\left.\left.X_{1}\right)\right)>1$ while that of $D F\left(X_{2}\right), \lambda_{2}=\exp \left(p_{1}-q_{1} X_{2}\right)+\alpha<1$, this establishes (2). The proof of (3) is similar and is omitted. In System (1), each axis is $F$ - invariant and on each $i$ - axis the map $F$ is $f_{i}$, a one hump map. In [22 - 24], R. May proved that $f_{2}$ undergoes period-doubling bifurcations. Proceeding exactly as in $[22-24]$ one obtains that the map $f_{1}$ undergoes similar period-doubling bifurcations. This completes the proof.

Now, we consider System (1) where both species have the same carrying capacity $\left(X_{1}=X_{2}\right)$.

Lemma 2: In System (1), let $X_{1}=X_{2}$. 


\section{F has a line of fixed points at $L$.}

2. If $p_{1}<\frac{2}{(1-\alpha)}+\ln (1-\alpha)$ and $p_{2}<2$, then $x_{1}>0$ or $x_{2}>0$ at the point $\left(x_{1}, x_{2}\right)$ in $R_{+}^{2}$ implies that $\omega\left(\left(x_{1}, x_{2}\right)\right) \subset L$.

Proof: Clearly, $X_{1}=X_{2}$ implies System (1) has a line of fixed points at $L$. Consider the nonzero point $\left(x_{1}, x_{2}\right)$ in $\mathbb{R}_{+}^{2}$. On the $i-$ axis, $X_{i}$ is locally stable implies it is globally stable in $(0, \infty)[9]$. Consequently, $p_{1}<\frac{2}{(1-\alpha)}+\ln (1-\alpha)$ implies $\omega\left(\left(x_{1}, 0\right)\right)=X_{1} \subset L$ while $p_{2}<2$ implies $\omega\left(\left(0, x_{2}\right)\right)=X_{2} \subset L$. Now, we consider the interior point $\left(x_{1}, x_{2}\right)$ with its entire orbit in the region below the line $L, B=\left\{\left(x_{1}, x_{2}\right) \in[0, \infty) \times[0, \infty) \mid x_{1}+x_{2}<X_{1}\right\}$. Then for each $i \in\{1,2\}$, $\left\{F_{i}^{t}\left(x_{1}, x_{2}\right)\right\}$ is an increasing sequence bounded above by the line segment $L$ in $\mathbb{R}_{+}^{2}$, and, $F^{t}\left(x_{1}, x_{2}\right)$ converges to a fixed point of $F$ on the line $L$. Also, if the entire orbit of $\left(x_{1}, x_{2}\right)$ is in the region above the line $L, A=\left\{\left(x_{1}, x_{2}\right) \in[0, \infty) \times[0, \infty) \mid\right.$ $\left.x_{1}+x_{2}>X_{1}\right\}$, then $\left\{F_{i}^{t}\left(x_{1}, x_{2}\right)\right\}$, a decreasing sequence bounded below by the line segment $L$ in $\mathbb{R}_{+}^{2}$, converges to a fixed point of $F$ on the line $L$.

Now we consider a positive point $\left(x_{1}, x_{2}\right)$ with some iterate in both regions $A$ and $B$. If there exists a positive integer $T$ such that $F^{t}\left(x_{1}, x_{2}\right)$ remains in $A$ (or $B$ ) for all $t \geq T$ then proceeding exactly as before, we obtain convergence to the line segment $L$. If the positive point $\left(x_{1}, x_{2}\right)$ is mapped in and out of region $A$ (or $B$ ) indefinitely, then there exists a subsequence $\left\{t_{i}\right\}$ with $t_{1}<t_{2}<$ $t_{3}<\ldots$ such that each $F^{t_{i}}\left(x_{1}, x_{2}\right) \in B$. In [9], Franke and Yakubu proved that $F_{2}^{t_{1}}\left(x_{1}, x_{2}\right)<F_{2}^{t_{2}}\left(x_{1}, x_{2}\right)<F_{2}^{t_{3}}\left(x_{1}, x_{2}\right)<\ldots$ Consequently, the continuity of $F$ and the boundedness of orbits force convergence to the line segment $L$.

Lemma 3: If a point $\left(x_{1}, x_{2}\right)$ in $\mathbb{R}_{+}^{2}$ is not in the basin of attraction of any fixed point, then there exist nonnegative integers $t_{1}, t_{2}$ and $T$ such that $F^{t_{1}}\left(x_{1}, x_{2}\right)$ $\in A$ and $F^{t_{2}}\left(x_{1}, x_{2}\right) \in B$, where $t_{1}, t_{2}>T$.

Proof: We will prove the result for region $B$. The proof for $A$ is similar and is omitted. Recall that the first quadrant is $F$ invariant. Suppose there exists a nonnegative integer $T$ such that the orbit of $F^{T}\left(x_{1}, x_{2}\right)$ does not intersect region $B$. 
Then the sequence of first coordinates, $F_{1}^{T}\left(x_{1}, x_{2}\right), F_{1}\left(F^{T}\left(x_{1}, x_{2}\right)\right), F_{1}^{2}\left(F^{T}\left(x_{1}, x_{2}\right)\right), \ldots$, is a nonincreasing sequence bounded below by zero and hence has a limit denoted by $\bar{x}_{1}$. Therefore, $F_{1}^{t}\left(x_{1}, x_{2}\right)-F_{1}^{t+1}\left(x_{1}, x_{2}\right) \rightarrow 0$ as $t \rightarrow \infty$. Hence, $F_{1}^{t}\left(x_{1}, x_{2}\right)+F_{2}^{t}\left(x_{1}, x_{2}\right) \rightarrow X_{1}$ as $t \rightarrow \infty$. This implies that $F_{2}^{t}\left(x_{1}, x_{2}\right) \rightarrow X_{1}-$ $\bar{x}_{1}$ as $t \rightarrow \infty$ and $F^{t}\left(x_{1}, x_{2}\right)$ is in the basin of attraction of a fixed point, a contradiction.

The following result (Corollary 1) is an immediate consequence of Lemma 3. Corollary 1:

1. $n$-cycles of $F$ in $R_{+}^{2}$ have some points in region $A$ and some in $B$ where $n \geq 2$.

2. 2 - cycles of $F$ in $R_{+}^{2}$ have one point in region $A$ and the other point in $B$.

\section{2-Cycles of $F$ (Yakubu's conjecture)}

System (1) has no isolated positive fixed points. However, on solving the system of two equations $x_{1}(t+2)=x_{1}(t)$ and $x_{2}(t+2)=x_{2}(t)$ simultaneously where $x_{1}(t), x_{2}(t) \neq 0$ we obtain that System (1) has a 2-cycle at

$$
\left(\begin{array}{c}
\frac{2 p_{2}-\gamma q_{2}\left(1+\exp \left(p_{2}-\gamma q_{2}\right)\right)}{q_{2}\left(\alpha+\exp \left(p_{1}-\gamma q_{1}\right)+\exp \left(p_{2}-\gamma q_{2}\right)\right)} \\
\gamma-\left(\frac{2 p_{2}-\gamma q_{2}\left(1+\exp \left(p_{2}-\gamma q_{2}\right)\right)}{q_{2}\left(\alpha+\exp \left(p_{1}-\gamma q_{1}\right)+\exp \left(p_{2}-\gamma q_{2}\right)\right)}\right)
\end{array}\right)
$$

where $\gamma=\frac{1}{q_{1}} \ln \left(\frac{-\beta \pm \sqrt{\left(\beta^{2}-4 \delta c\right)}}{2 \delta}\right), \beta=\alpha^{2}-1+\exp \left(2\left(p_{1}-p_{2} \frac{q_{1}}{q_{2}}\right)\right), \delta=\alpha \exp \left(p_{1}-2 p_{2} \frac{q_{1}}{q_{2}}\right)$ and $c=\alpha \exp \left(p_{1}\right)[28,29]$. Yakubu conjectured, in [29], that if $p_{1}=\frac{3}{2}, q_{1}=$ $1, q_{2}=1$ and $\alpha=\frac{1}{2}$ then System (1) has a globally stable positive 2-cycle in $(0, \infty) \times(0, \infty)$ whenever $X_{1} \leq p_{2} \leq 3.411822071$. Here, we use a very general result to show that in connected metric spaces, $n$-cycles of continuous maps are not globally stable whenever $n \geq 2$. Consequently, in Yakubu's conjecture, the positive $2-$ cycle is locally asymptotically stable and not globally stable. 
First, we introduce additional notation and definitions. Let $f: \mathbb{X} \rightarrow \mathbb{X}$ be a continuous map on a metric space $\mathbb{X}$ and $x^{*} \in \mathbb{X}$ is a locally asymptotically stable fixed point of $f$. The basin of attraction of $x^{*}, W_{f}^{S}\left(x^{*}\right)$, is $\left\{x \in \mathbb{X} \mid \lim _{n \rightarrow \infty} f^{n}(x)=\right.$ $\left.x^{*}\right\}$. If $y^{*} \in \mathbb{X}$ is a locally asymptotically stable k-periodic point of $f$ then it is a fixed poinnt of $g=f^{k}$ and its basin of attraction under $\mathrm{g}$ is $\mathrm{W}_{g}^{S}\left(y^{*}\right)$. The basin of attraction of the cycle $O\left(\mathrm{y}^{*}\right)=\left(\mathrm{y}^{*}, f\left(y^{*}, \ldots, f(k-1)\left(y^{*}\right)\right.\right.$ is given by $W^{S}\left(O\left(y^{*}\right)\right)=$ $\cup_{j=0}^{k-1} W_{g}^{S}\left(f^{j}\left(y^{*}\right)\right)$.

$W_{f}^{S}\left(x^{*}\right)$ is an open $f$ invariant set [6]. Furthermore, the complement of $W_{f}^{S}\left(x^{*}\right)$ in $\mathbb{X}$ and the boundary of $W_{f}^{S}\left(x^{*}\right)$ in $\mathbb{X}$ are $f$ invariant sets [6. Nex$\mathrm{t}$, we prove that stable period $k$-cycles are not globally stable in connected metric spaces.

Theorem 3. If $f: X \rightarrow X$ is a continuous map on a connected metric space $X$ and $y^{*} \in X$ is a locally asymptotically stable $k$-cycle of $f$ with $k \geq 2$, then $W^{S}\left(O\left(y^{*}\right)\right) \varsubsetneqq X$.

Proof: For each $j \in\{0,1,2, \ldots, k-1\}, W_{g}^{S}\left(f^{j}\left(y^{*}\right)\right)$ is a nonempty subset of $\mathbb{X}$ and contains $f^{j}\left(y^{*}\right)$, since $g\left(f^{j}\left(y^{*}\right)\right)=f^{j}\left(y^{*}\right)$. Furthermore, the sets $W_{g}^{S}\left(y^{*}\right)$, $W_{g}^{S}\left(f\left(y^{*}\right)\right), \ldots, W_{g}^{S}\left(f^{k-1}\left(y^{*}\right)\right)$ are disjoint. If $W^{S}\left(O\left(y^{*}\right)\right)=\mathbb{X}$, then $\mathbb{X}$ is a disjoint union of nonempty open sets. This is impossible as $\mathbb{X}$ is a connected metric space.

\subsection{Example}

In Yakubu's example with $p_{1}=\frac{3}{2}, q_{1}=1, q_{2}=1, \alpha=\frac{1}{2}$ and $p_{2}=2.2$, System (1) has a locally asymptotically stable positive $2-$ cycle at

$$
\left\{\left(\begin{array}{l}
0.4063 \\
1.9592
\end{array}\right),\left(\begin{array}{l}
0.3741 \\
1.6604
\end{array}\right)\right\} \text {. }
$$

The positive 2 - cycle is locally asymptotically stable and not globally stable in $(0, \infty) \times(0, \infty)$ (Theorem 3$)$. In fact, $X_{2}$ is a saddle fixed-point and $X_{1}=$ $1.5+\ln 2<X_{2}=2.2$ implies that the stable manifold of $X_{2}$ is in the interior of $R_{+}^{2}$ (Lemma 1) while the unstable manifold is on the vertical axis. Figure 1 shows the stable manifold of $X_{2}$ (black region in $\left.(0, \infty) \times(0, \infty)\right)$ and the basin 
of attraction of the positive $2-$ cycle (white region in $(0, \infty) \times(0, \infty)$ ). A lot of positive population sizes are in the stable manifold of the saddle fixed-point, $X_{2}=2.8$, and a lot more are in the basin of attraction of the positive 2 -cycle(see Figure 1). FIG. 1: Stable manifold of $X_{2}$ (black region) and basin of attraction of the positive $2-\operatorname{cycle}$ (white region). Horizontal axis is $x_{1}$ and vertical axis is $x_{2}$. The parameter $p_{2}$ measures the level of intraspecific competition in Species 2 . To study the changes in the basin of attraction of the positive 2 - cycle as we increase the level of intraspecific competition, we keep the parameters $p_{1}, q_{1}, q_{2}$,

and $\alpha$ fixed at $p_{1}=\frac{3}{2}, q_{1}=1, q_{2}=1$ and $\alpha=\frac{1}{2}$ and increase $p_{2}$. At $p_{2}=3$, the system has a locally asymptotically stable positive 2 - cycle at

$$
\left\{\left(\begin{array}{l}
0.5793 \\
0.6103
\end{array}\right),\left(\begin{array}{l}
1.0799 \\
3.7305
\end{array}\right)\right\}
$$

coexisting with the saddle fixed-point at $X_{2}=3$. Figure 2 shows the stable manifold of $X_{2}$ (black region in $\left.(0, \infty) \times(0, \infty)\right)$ and the basin of attraction of the positive 2 -cycle (white region in $(0, \infty) \times(0, \infty))$. Our numerical simulations show an increase in fragmentation of the basin of attraction of the 2 -cycle as we increase $p_{2}$. Constructing the geometric structure of the basin of attraction of the 2-cycle and deciding if it is a connected set are open questions that we are working on.FIG. 2: Stable manifold of $X_{2}$ (black region) and basin of attraction of the positive $2-c y c l e\left(\right.$ white region). Horizontal axis is $x_{1}$ and vertical axis is $x_{2}$.

\section{$5 \quad$ References}

1. M. Begon, J. L. Harper and C. R. Townsend, Ecology: Individuals, Populations and Communities, 3rd Edition. Blackwell Science, Inc. Cambridge, MA, 1996.

2. F. Brauer and A. A. Sanchez, Constant rate population harvesting: Equilibrium and stability, Theor. Popl. Biol. 8:12-30 (1975). 
3. P. Collet and J.- P. Eckmann, Iterated Maps of the Interval as Dynamical Systems, Birkhäuser, Boston, 1980.

4. H. N. Comins and M. P. Hassell, Predation in multiprey communities, J. Theor. Biol. 62: 93-114 (1976).

5. R. L. Devaney, An Introduction to Chaotic Dynamical Systems, Benjamin/Cummings, Menlo Park, CA, 1986.

6. S. N. Elaydi, Discrete Chaos. Chapman \& Hall/CRC, Boca Raton, FL, 2000 .

7. S. N. Elaydi, An Introduction to Difference Equations. 2nd Edition. Undergraduate Texts in Mathematics, Springer-Verlag, New York, 1999.

8. A. Fonda, Uniformly persistent semi-dynamical systems, Proc. Amer. Math. Soc. 104:111-116 (1988).

9. J. E. Franke and A.-A. Yakubu, Global attractors in competitive systems, Nonlinear Anal. Theor. Methods Appl. 16:111-129 (1991).

10. J. E. Franke and A.-A. Yakubu, Mutual exclusion versus coexistence for discrete competitive systems, J. Math. Biol. 30:161-168 (1991).

11. J. E. Franke and A.-A. Yakubu, Pioneer exclusion in a one hump discrete pioneer-climax competitive system, J. Math. Biol. 32:771-787 (1994).

12. H. I. Freedman and J. W.-H. So, Uniform persistence and repellors for maps, AMS Proc. 107:1137-1142 (1989).

13. T. Hallam and S. Levin, Mathematical Ecology: An introduction, Biomathematics 17, Springer-Verlag, 1986.

14. B.-L. Hao, Chaos, World Scientific, Singapore, 1984. 
15. M. P. Hassell and H. N. Comins, Discrete time models for two-species competition, Theor. Pop. Biol. 9:202-221 (1976).

16. J. Hofbauer, V. Hutson and W. Jansen, Coexistence for systems governed by difference equations of Lotka-Volterra type, J. Math. Biol. 25:553-570 (1987).

17. H. Jiang and T. D. Rogers, The discrete dynamics of symmetric competition, J. Math. Biol. 15:573-596 (1987).

18. V. L. Kocic' and G. Ladas, Global Behavior of Nonlinear Difference Equations of Higher Order with Applications, Kluwer Academic, Boston, 1993.

19. T. Li and J. Yorke, Period-3 implies chaos, Amer. Math. Monthly 82:985998 (1975).

20. B. Mathews and N. Neumann, Persistence of Two Competing Species With Planting, Preprint.

21. B. Mathews and N. Neumann, An Attracting Two-Cycle In a Discrete Model Of Two Competing Species With Planting, Preprint.

22. R. M. May, Biological populations with nonoverlapping generations: Stable points, stable cycles, and chaos, Science 186: 645-647 (1974).

23. R. M. May, Biological populations obeying difference equations: Stable points, stable cycles, and chaos, J. Theor. Biol. 51:511-524 (1975).

24. R. M. May, Simple mathematical models with very complicated dynamics, Nature 261:459-467 (1976).

25. H. I. McCallum, Effects of immigration on chaotic population dynamics, $J$. Theor. Biol. 154: 277-284 (1992). 
26. J. F. Selgrade, Planting and harvesting for pioneer-climax models, Rocky Mountain J. Math. 24: 293-310 (1994).

27. J. F. Selgrade, Using stocking or harvesting to reverse period-doubling bifurcations in discrete population models, J. Diff. Equations Appl. 4: 163183 (1998).

28. A. A. Yakubu, The effects of planting and harvesting on endangered species in discrete competitive systems, Math. Biosci. 126:1-20 (1995).

29. A. A. Yakubu, A discrete competitive system with planting, J. Diff. Equations Appl. 4: 213-214 (1998). 